Available at: http://www.ictp.trieste.it/〜pub_off $\mathrm{IC} / 2000 / 83$

United Nations Educational Scientific and Cultural Organisation and

International Atomic Energy Agency

THE ABDUS SALAM INTERNATIONAL CENTRE FOR THEORETICAL PHYSICS

\title{
ORIENTING METHOD FOR OBSTACLE PROBLEMS
}

\author{
H. X. $\mathrm{Phu}^{1}$ \\ Institute of Mathematics, P.O. Box 631 Bo Ho, 10000 Hanoi, Vietnam \\ and \\ The Abdus Salam International Centre for Theoretical Physics, Trieste, Italy \\ and \\ T. D. Long \\ Department of Mathematics, Hue University, 27 Nguyen Hue Str., Hue, Vietnam.
}

\begin{abstract}
This paper deals with the obstacle problem

$$
\begin{array}{ll}
\operatorname{minimize} & \int_{\Omega} F(v) d x \\
\text { subject to } & v \geq r \text { in } \Omega, \quad v=g \text { on } \partial \Omega,
\end{array}
$$
\end{abstract}

where $\Omega \subset \mathbb{R}^{n}, F$ is a suitable functional, and $r$ and $g$ are given functions. Some sufficient criteria are stated for determining parts of the coincidence set $\mathcal{C}(u)=$ $\{x \in \Omega: u(x)=r(x)\}$ and the noncoincidence set $\mathcal{N}(u)=\{x \in \Omega: u(x)>r(x)\}$ of the optimal solution $u$ to the above obstacle problem.

$$
\text { MIRAMARE - TRIESTE }
$$

July 2000

\footnotetext{
1 Regular Associate of the Abdus Salam ICTP.
} 


\section{Introduction}

Numerous mathematical and physical problems can be formulated as the minimum problem

$$
\text { minimize } \int_{\Omega} F(v) d x \quad \text { subject to } \quad v \in \mathcal{K},
$$

where $\Omega$ is a domain in $\mathbb{R}^{n}$. For instance, the well known Dirichlet problem reads as follows:

$$
\text { minimize } \int_{\Omega}|\nabla v|^{2} d x \quad \text { subject to } v=g \text { on } \partial \Omega,
$$

where $|\cdot|$ denotes the Euclidean norm. The corresponding Euler equation for its optimal solution $u$ is the boundary value problem

$$
\triangle u=0 \text { in } \Omega, \quad u=g \text { on } \partial \Omega
$$

(see e.g. [16]). If the state function $v$ has to satisfy an additional restriction like $v \geq r$, there arises a so-called obstacle problem

$$
\begin{array}{ll}
\operatorname{minimize} & \int_{\Omega}|\nabla v|^{2} d x \\
\text { subject to } & v \in \mathcal{K}:=\{v \in \mathcal{V}: v \geq r \text { in } \Omega, \quad v=g \text { on } \partial \Omega\},
\end{array}
$$

where $\mathcal{V}$ stands for some suitable vector space of functions. Because of the restriction $v \geq r$, the obstacle problem (1.2) does not lead to the boundary value problem (1.1), but to the variational inequality

$$
u \in \mathcal{K}: \int_{\Omega} \nabla u \cdot \nabla(v-u) d x \geq 0 \text { for all } v \in \mathcal{K}
$$

(see $[15$, p. 3]). Such a problem is more complicated than (1.1). In fact, the partial differential equation $\triangle u=0$ is still valid in the so-called noncoincidence set

$$
\mathcal{N}(u):=\{x \in \Omega: u(x)>r(x)\} .
$$

Since $u$ is determined in the coincidence set

$$
\mathcal{C}(u):=\{x \in \Omega: u(x)=r(x)\}=\Omega \backslash \mathcal{N}(u),
$$

under the continuity assumption of first derivatives, one still has to consider the remaining problem

$$
\begin{aligned}
& \triangle u=0 \text { in } \mathcal{N}(u) \\
& u=g \text { on } \partial \Omega \cap \partial \mathcal{N}(u), \\
& u=r \text { and } \frac{\partial u}{\partial n}=\frac{\partial r}{\partial n} \text { on } \Omega \cap \partial \mathcal{N}(u) .
\end{aligned}
$$


Since $\Omega \cap \partial \mathcal{N}(u)$ is not known a priori, it is called a free boundary (see [15, p. 5]). This notation may cause misunderstanding for strangers. In concrete examples, it is by nature not free at all, but already fixed by the given problem statement. The only problem is that one does not know something about it a priori.

Here, "a priori" normally means "before solving (1.2) or (1.3) or (1.4)". But in this paper we state some sufficient criteria for determining parts of coincidence and noncoincidence sets $\mathcal{C}(u)$ and $\mathcal{N}(u)$, without solving the original obstacle problem (1.2), or the variational inequality (1.3), or its corresponding free boundary problem (1.4).

The idea originated from the so-called Method of Orienting Curves which was developed in [3], [4], [9], [12], and [14] for solving optimal control problems with state constraints. Its application area consists of problems with ordinary differential equations (i.e. with one independent variable) having one state function. Although this area is rather narrow, we had successfully applied this method for solving some relevant problems, such as constrained Zermelo's navigation problem [10], Steiner's problem of finding an inpolygon of some given convex polygon with minimal circumference [11], inventory problem [12], optimal control of hydroelectric power plants [5], and robot motion along a prescribed trajectory [13]. By this method, following so-called orienting curves, optimal trajectories are constructed part by part.

In this paper, we investigate problems with several independent variables. Thus surfaces appear instead of curves. Therefore, the shortened name "Orienting Method" is more appropriate. It is understandable if we cannot obtain such a complete result as in case with one independent variable. But in a similar way, barrier functions and bottle neck points can be used to locate some coincidence and noncoincidence points of optimal solutions.

In Section 2, we state the concrete formulation and some examples of the problem class considered. Section 3 contains sufficient criteria for coincidence and noncoincidence points under a uniqueness assumption. Section 4 is devoted a special case where some invariance assumption is made.

\section{Problem Statement}

Let us first introduce some notations. For $\Omega$ as some domain (i.e. a connected open subset) in $\mathbb{R}^{n}, \mathcal{V}(\Omega)$ denotes some family of suitable vector spaces of realvalued functions on $\Omega$ satisfying:

$\left(\mathcal{V}_{1}\right) v \in \mathcal{V}(\Omega)$ is continuous on $\bar{\Omega}$;

$\left(\mathcal{V}_{2}\right)$ If $v \in \mathcal{V}(\Omega)$ and $\Omega^{\prime} \subset \Omega$ then the restriction $\left.v\right|_{\Omega^{\prime}}$ of $v$ on $\Omega^{\prime}$ belongs to $\mathcal{V}\left(\Omega^{\prime}\right)$ 
$\left(\mathcal{V}_{3}\right)$ If $v \in \mathcal{V}(\Omega), v^{\prime} \in \mathcal{V}\left(\Omega^{\prime}\right), \Omega^{\prime} \subset \Omega$, and $v=v^{\prime}$ on $\partial \Omega^{\prime}$ then $\tilde{v}$ defined by

$$
\tilde{v}(x)= \begin{cases}v^{\prime}(x) & \text { if } x \in \Omega^{\prime} \\ v(x) & \text { if } x \in \Omega \backslash \Omega^{\prime}\end{cases}
$$

belongs to $\mathcal{V}(\Omega)$.

For instance, $\mathcal{V}(\Omega)=C(\bar{\Omega})$ fulfills the above three conditions, but $\mathcal{V}(\Omega)=$ $C^{1}(\bar{\Omega})$ violates $\left(\mathcal{V}_{3}\right)$.

Let

$$
F: \mathcal{V}(\Omega) \rightarrow L^{1}(\Omega)
$$

that means $\mathcal{V}(\Omega)$ and $F$ are chosen so that the integral $\int_{\Omega} F(v) d x$ is finitely defined.

For some suitable functions $g$ and $r$, denote

$$
\mathcal{K}_{r}^{\Omega, g}:=\{v \in \mathcal{V}(\Omega): v \geq r \text { in } \Omega, \quad v=g \text { on } \partial \Omega\}
$$

Now, for fixed $\Omega \subset \mathbb{R}^{n}, g$ and $r$, consider the following minimum problem

$$
\text { minimize } \mathcal{F}^{\Omega}(v):=\int_{\Omega} F(v) d x \quad \text { subject to } \quad v \in \mathcal{K}_{r}^{\Omega, g}
$$

Our goal is to determine some parts of the coincidence set

$$
\mathcal{C}\left(u_{r}^{\Omega, g}\right)=\left\{x \in \Omega: u_{r}^{\Omega, g}(x)=r(x)\right\}
$$

and the noncoincidence set

$$
\mathcal{N}\left(u_{r}^{\Omega, g}\right)=\left\{x \in \Omega: u_{r}^{\Omega, g}(x)>r(x)\right\}
$$

of the optimal solution $u_{r}^{\Omega, g}$ to Problem $\left(\mathcal{P}_{r}^{\Omega, g}\right)$ (whose elements are called coincidence or noncoincidence points, respectively).

To avoid difficulties caused by the obstacle $v \geq r$ we do not deal directly with the obstacle problem $\left(\mathcal{P}_{r}^{\Omega, g}\right)$, but investigate corresponding minimum problems without obstacle

$$
\text { minimize } \quad \mathcal{F}^{\Omega^{\prime}}(v)=\int_{\Omega^{\prime}} F(v) d x \quad \text { subject to } \quad v \in \mathcal{K}^{\Omega^{\prime}, g^{\prime},} \quad\left(\mathcal{P}^{\Omega^{\prime}, g^{\prime}}\right)
$$

where $\Omega^{\prime}$ is some subdomain of $\Omega, g^{\prime}$ is some suitable function on $\partial \Omega^{\prime}$, and

$$
\mathcal{K}^{\Omega^{\prime}, g^{\prime}}:=\left\{v \in \mathcal{V}\left(\Omega^{\prime}\right): v=g^{\prime} \text { on } \partial \Omega^{\prime}\right\}
$$

These problems are complicated enough, but they are easier than the original one.

The most essential assumption throughout this paper is concerned with the uniqueness of optimal solutions to problems without obstacle, namely: 
$\left(\mathcal{A}_{U}\right)$ For all $\Omega^{\prime} \subset \Omega$ and $g^{\prime} \in C\left(\partial \Omega^{\prime}\right)$, the corresponding problem $\left(\mathcal{P}^{\Omega^{\prime}, g^{\prime}}\right)$ admits at most one optimal solution. More precisely, if

$$
u_{1}, u_{2} \in \mathcal{K}^{\Omega^{\prime}, g^{\prime}} \text { and } \mathcal{F}^{\Omega^{\prime}}\left(u_{1}\right)=\mathcal{F}^{\Omega^{\prime}}\left(u_{2}\right)=\inf _{v \in \mathcal{K}^{\Omega^{\prime}, g^{\prime}}} \mathcal{F}^{\Omega^{\prime}}(v)
$$

then

$$
u_{1}=u_{2} \text { in } \Omega^{\prime}
$$

Numerous relevant obstacle problems belong to class $\left(\mathcal{P}_{r}^{\Omega, g}\right)$ and fulfill $\left(\mathcal{A}_{U}\right)$ as the following examples show.

Example 2.1. Consider the obstacle Dirichlet problem

$$
\text { minimize } \mathcal{F}_{1}^{\Omega}(v):=\int_{\Omega}|\nabla v|^{2} d x
$$

subject to $\quad v \in \mathcal{K}_{r}^{\Omega, g}=\{v \in \mathcal{V}(\Omega): v \geq r$ in $\Omega, \quad v=g$ on $\partial \Omega\}$.

For

$$
\mathcal{V}(\Omega)=W^{1,2}(\Omega) \cap C(\bar{\Omega})
$$

$\left(\mathcal{V}_{1}\right)-\left(\mathcal{V}_{3}\right)$ are fulfilled, and $\mathcal{F}_{1}^{\Omega}$ is well defined. Now we show that $\mathcal{F}_{1}^{\Omega^{\prime}}$ is strictly convex on

$$
\mathcal{K}^{\Omega^{\prime}, g^{\prime}}=\left\{v \in \mathcal{V}\left(\Omega^{\prime}\right): v=g^{\prime} \text { on } \partial \Omega^{\prime}\right\}
$$

For $0<\lambda<1, \mu=1-\lambda$, and $v, u \in \mathcal{K}^{\Omega, g}$, it holds

$$
\begin{aligned}
& \lambda \mathcal{F}_{1}^{\Omega^{\prime}}(u)+\mu \mathcal{F}_{1}^{\Omega^{\prime}}(v)-\mathcal{F}_{1}^{\Omega^{\prime}}(\lambda u+\mu v) \\
& =\int_{\Omega^{\prime}}\left(\lambda|\nabla u|^{2}+\mu|\nabla v|^{2}-|\lambda \nabla u+\mu \nabla v|^{2}\right) d x \\
& =\int_{\Omega^{\prime}}\left(\left(\lambda-\lambda^{2}\right)|\nabla u|^{2}+\left(\mu-\mu^{2}\right)|\nabla v|^{2}-2 \lambda \mu(\nabla u \mid \nabla v)\right) d x \\
& =\lambda \mu \int_{\Omega^{\prime}}|\nabla u-\nabla v|^{2} d x \\
& \geq 0 .
\end{aligned}
$$

Hence $\mathcal{F}_{1}^{\Omega^{\prime}}$ is convex. Moreover,

$$
\lambda \mathcal{F}_{1}^{\Omega^{\prime}}(u)+\mu \mathcal{F}_{1}^{\Omega^{\prime}}(v)-\mathcal{F}_{1}^{\Omega^{\prime}}(\lambda u+\mu v)=0
$$

if and only if

$$
\|\nabla(u-v)\|_{L^{2}\left(\Omega^{\prime}\right)}^{2}=\int_{\Omega^{\prime}}|\nabla(u-v)|^{2} d x=0 .
$$

Since $u=v=g^{\prime}$ on $\partial \Omega^{\prime}$, Poincaré inequality (see [2, p. 26]) implies

$$
\|u-v\|_{L^{2}\left(\Omega^{\prime}\right)} \leq K\|\nabla(u-v)\|_{L^{2}\left(\Omega^{\prime}\right)}
$$


for some $K>0$. Therefore, (2.5) implies $\|u-v\|_{L^{2}\left(\Omega^{\prime}\right)}=0$ and

$$
\|u-v\|_{W^{1,2}\left(\Omega^{\prime}\right)}=\left(\|u-v\|_{L^{2}\left(\Omega^{\prime}\right)}^{2}+\|\nabla(u-v)\|_{L^{2}\left(\Omega^{\prime}\right)}^{2}\right)^{1 / 2}=0
$$

This means that $\mathcal{F}_{1}^{\Omega^{\prime}}$ is strictly convex on $\mathcal{K}^{\Omega^{\prime}, g^{\prime}}$. Consequently, $\left(\mathcal{P}^{\Omega^{\prime}, g^{\prime}}\right)$ admits at most one optimal solution, i.e. $\left(\mathcal{A}_{U}\right)$ is satisfied.

Example 2.2. Problems of minimal surfaces have been studied by many mathematicians (see [1] and [8], for instance). Here we only investigate a special case with an obstacle which can be stated in the form

$$
\begin{array}{ll}
\operatorname{minimize} & \mathcal{F}_{2}^{\Omega}(v):=\int_{\Omega} \sqrt{1+|\nabla v|^{2}} d x \\
\text { subject to } & v \in \mathcal{K}_{r}^{\Omega, g}=\{v \in \mathcal{V}(\Omega): v \geq r \text { in } \Omega, \quad v=g \text { on } \partial \Omega\}
\end{array}
$$

(see $\left[15\right.$, p. 9]). Let us prove that $\mathcal{F}_{2}^{\Omega^{\prime}}$ is strictly convex on $\mathcal{K}^{\Omega^{\prime}, g^{\prime}}$ which is defined like in Example 2.1.

For $0<\lambda<1, \mu=1-\lambda, v, u \in \mathcal{K}^{\Omega, g}$, and

$$
\left.D:=\left(\lambda \sqrt{1+|\nabla u|^{2}}+\mu \sqrt{1+|\nabla v|^{2}}\right)^{2}-\left(\sqrt{1+|\lambda \nabla u+\mu \nabla v|^{2}}\right)\right)^{2}
$$

it follows from Schwarz inequality $([16, \mathrm{p} .8])$ that

$$
\begin{aligned}
D & =2 \lambda \mu\left(\sqrt{\left(1+|\nabla u|^{2}\right)\left(1+|\nabla v|^{2}\right)}-(\nabla u \mid \nabla v)-1\right) \\
& =2 \lambda \mu\left(\sqrt{(1+|\nabla u||\nabla v|)^{2}+(|\nabla u|-|\nabla v|)^{2}}-(\nabla u \mid \nabla v)-1\right) \\
& \geq 2 \lambda \mu((1+|\nabla u||\nabla v|)-(\nabla u \mid \nabla v)-1) \\
& \geq 0 .
\end{aligned}
$$

This is equivalent to

$$
\lambda \sqrt{1+|\nabla u|^{2}}+\mu \sqrt{1+|\nabla v|^{2}} \geq \sqrt{1+|\lambda \nabla u+\mu \nabla v|^{2}}
$$

where the equality holds if and only if

$$
|\nabla u|=|\nabla v| \text { and } \quad \nabla u=\alpha \nabla v \text { for some } \alpha \geq 0
$$

which yields obviously

$$
\nabla u=\nabla v
$$


Applying this result we have

$$
\lambda \mathcal{F}_{2}^{\Omega^{\prime}}(u)+\mu \mathcal{F}_{2}^{\Omega^{\prime}}(v)-\mathcal{F}_{2}^{\Omega^{\prime}}(\lambda u+\mu v)=\int_{\Omega^{\prime}} D(x) d x \geq 0
$$

where the equality holds only if

$$
\|\nabla(u-v)\|_{L^{2}\left(\Omega^{\prime}\right)}^{2}=\int_{\Omega^{\prime}}|\nabla(u-v)|^{2} d x=0 .
$$

This is just the same situation as in Example 2.1. Hence, $\mathcal{F}_{2}^{\Omega^{\prime}}$ is strictly convex on $\mathcal{K}^{\Omega^{\prime}, g^{\prime}}$, and $\left(\mathcal{A}_{U}\right)$ is satisfied.

Example 2.3. Consider now the deformation of a membrane constrained by an obstacle. As in [15, pp. 1-2], the problem of finding the equilibrium position of such a membrane can be stated as follows:

$$
\begin{array}{ll}
\operatorname{minimize} & \mathcal{F}_{3}^{\Omega}(v):=\mathcal{F}_{4}^{\Omega}(v)-\mathcal{F}_{5}^{\Omega}(v) \\
\text { subject to } & v \in \mathcal{K}_{r}^{\Omega, g}=\{v \in \mathcal{V}(\Omega): v \geq r \text { in } \Omega, \quad v=g \text { on } \partial \Omega\}
\end{array}
$$

where $\mathcal{F}_{4}^{\Omega}(v)$ is the potential energy of the deformed membrane, which is assumed to be proportional to the increase of membrane surface, i.e.

$$
\mathcal{F}_{4}^{\Omega}(v)=\sigma \int_{\Omega} \sqrt{1+|\nabla v|^{2}} d x \quad \text { for some constant } \sigma>0
$$

and

$$
\mathcal{F}_{5}^{\Omega}(v)=\int_{\Omega} f v d x
$$

describes the work done by a normal uniformly distributed external force given by $f$. Since $\mathcal{F}_{5}^{\Omega}$ is linear, and $\mathcal{F}_{4}^{\Omega}(v)=\sigma \mathcal{F}_{2}^{\Omega}(v)$, it follows from Example 2.2 that $\mathcal{F}_{3}^{\Omega^{\prime}}$ is strictly convex on $\mathcal{K}^{\Omega^{\prime}}, g^{\prime}$ (for all suitable $\Omega^{\prime}$ and $g^{\prime}$ ), and all assumptions $\left(\mathcal{V}_{1}\right)-\left(\mathcal{V}_{3}\right)$ and $\left(\mathcal{A}_{U}\right)$ are fulfilled by choosing $\mathcal{V}(\Omega)=W^{1,2}(\Omega) \cap C(\bar{\Omega})$.

Usually, $\mathcal{F}_{4}^{\Omega}(v)$ is approximated by $\mathcal{F}_{1}^{\Omega}(v)$, i.e.

$$
\mathcal{F}_{4}^{\Omega}(v)=\sigma \int_{\Omega}|\nabla v|^{2} d x
$$

(see $[15$, p. 2]). In this case, the desired conclusion can be deduced from the result of Example 2.1.

Example 2.4. According to [15, p. 7], the total potential energy of a bending plate can be written in the form

$$
\mathcal{F}_{6}^{\Omega}(v):=\frac{D}{2 h} \int_{\Omega}|\triangle v|^{2} d x+\frac{T}{2} \int_{\Omega}|\nabla v|^{2} d x-\int_{\Omega} f v d x
$$


where $\Omega \subset$ is a bounded domain in $\mathbb{R}^{2}, h>0$ is the thickness, $D>0$ is the stiffness coefficient of the plate, $T>0$ denotes the constant absolute value of stress per unit surface, and $f=f(x)$ represents a density of external forces per unit surface (compare [7, pp. 74-79]).

Let $\mathcal{V}(\Omega)=W^{2,2}(\Omega)$. Since the embedding $W^{2,2}(\Omega) \subset C(\bar{\Omega})$ is compact, a function $v \in W^{2,2}(\Omega)$ also belongs to $C(\bar{\Omega})$ provided we change $u$ on a suitable set of two-dimensional measure zero (see $\left[16\right.$, p. 1027]). Hence $\left(\mathcal{V}_{1}\right)-\left(\mathcal{V}_{3}\right)$ are fulfilled.

Let us justify $\left(\mathcal{A}_{U}\right)$. The functional

$$
\mathcal{F}_{7}^{\Omega}(v):=\int_{\Omega}|\triangle v|^{2} d x
$$

is convex, because it holds for $0<\lambda<1, \mu=1-\lambda$ and $v, u \in \mathcal{K}^{\Omega, g}$

$$
\lambda \mathcal{F}_{7}^{\Omega}(v)+\mu \mathcal{F}_{7}^{\Omega}(u)-\mathcal{F}_{7}^{\Omega}(\lambda v+\mu u)=\lambda \mu \int_{\Omega}|\triangle v-\triangle u|^{2} d x \geq 0 .
$$

By Example 2.1, the functional

$$
\mathcal{F}_{1}^{\Omega}(v)=\int_{\Omega}|\nabla v|^{2} d x
$$

is strictly convex on $W_{0}^{1,2}(\Omega)$. Since the embedding $W_{0}^{2,2}(\Omega) \subset W_{0}^{1,2}(\Omega)$ is compact (see $\left[16\right.$, p. 1027]), $\mathcal{F}_{1}^{\Omega}$ is also strictly convex on $W_{0}^{2,2}(\Omega)$. Hence, the functional

$$
\mathcal{F}_{6}^{\Omega}(v)=\frac{D}{2 h} \mathcal{F}_{7}^{\Omega}(v)+\frac{T}{2} \mathcal{F}_{1}^{\Omega}(v)-\int_{\Omega} f v d x
$$

is strictly convex on

$$
\mathcal{K}^{\Omega, g}=\left\{v \in W^{2,2}(\Omega): v=g \text { on } \partial \Omega\right\}
$$

that means $\left(\mathcal{A}_{U}\right)$ is satisfied.

We have seen that there are numerous relevant problems which fulfill the assumptions stated above.

\section{Main Results}

As first consequence of assumption $\left(\mathcal{A}_{U}\right)$, we have the following local optimal property. 
Proposition 3.1. Let $\Omega^{\prime \prime} \subset \Omega^{\prime} \subset \Omega$ and $u^{\Omega^{\prime}, g^{\prime}}$ be optimal to $\left(\mathcal{P}^{\Omega^{\prime}, g^{\prime}}\right)$. Then the restriction $\left.u^{\Omega^{\prime}, g^{\prime}}\right|_{\Omega^{\prime \prime}}$ of $u^{\Omega^{\prime}, g^{\prime}}$ on $\Omega^{\prime \prime}$ is the unique optimal solution to $\left(\mathcal{P}^{\Omega^{\prime \prime}}, g^{\prime \prime}\right)$ where $g^{\prime \prime}$ is the restriction $\left.u^{\Omega^{\prime}, g^{\prime}}\right|_{\partial \Omega^{\prime \prime}}$ of $u^{\Omega^{\prime}, g^{\prime}}$ on $\partial \Omega^{\prime \prime}$.

Proof. By $\left(\mathcal{V}_{2}\right),\left.u^{\Omega^{\prime}, g^{\prime}}\right|_{\Omega^{\prime \prime}} \in \mathcal{V}\left(\Omega^{\prime \prime}\right)$, and therefore, by definition,

$$
\left.u^{\Omega^{\prime}, g^{\prime}}\right|_{\Omega^{\prime \prime}} \in \mathcal{K}^{\Omega^{\prime \prime}, g^{\prime \prime}} .
$$

Assume the contrary that $\left(\mathcal{P}^{\Omega^{\prime \prime}, g^{\prime \prime}}\right)$ has $u \in \mathcal{K}^{\Omega^{\prime \prime}, g^{\prime \prime}}$ as a better solution, i.e.

$$
\mathcal{F}^{\Omega^{\prime \prime}}(u)<\mathcal{F}^{\Omega^{\prime \prime}}\left(\left.u^{\Omega^{\prime}, g^{\prime}}\right|_{\Omega^{\prime \prime}}\right) .
$$

Consider

$$
\tilde{u}(x):= \begin{cases}u(x) & \text { if } x \in \Omega^{\prime \prime} \\ u^{\Omega^{\prime}, g^{\prime}}(x) & \text { if } x \in \Omega^{\prime} \backslash \Omega^{\prime \prime}\end{cases}
$$

By $\left(\mathcal{V}_{3}\right), \tilde{u} \in \mathcal{V}\left(\Omega^{\prime}\right)$ and therefore $\tilde{u} \in \mathcal{K}^{\Omega^{\prime}, g^{\prime}}$. Furthermore

$$
\begin{aligned}
0 & >\mathcal{F}^{\Omega^{\prime \prime}}(u)-\mathcal{F}^{\Omega^{\prime \prime}}\left(\left.u^{\Omega^{\prime}, g^{\prime}}\right|_{\Omega^{\prime \prime}}\right) \\
& =\mathcal{F}^{\Omega^{\prime \prime}}(\tilde{u})+\mathcal{F}^{\Omega^{\prime} \backslash \Omega^{\prime \prime}}(\tilde{u})-\mathcal{F}^{\Omega^{\prime} \backslash \Omega^{\prime \prime}}\left(u^{\Omega^{\prime}, g^{\prime}}\right)-\mathcal{F}^{\Omega^{\prime \prime}}\left(\left.u^{\Omega^{\prime}, g^{\prime}}\right|_{\Omega^{\prime \prime}}\right) \\
& =\mathcal{F}^{\Omega^{\prime}}(\tilde{u})-\mathcal{F}^{\Omega^{\prime}}\left(u^{\Omega^{\prime}, g^{\prime}}\right),
\end{aligned}
$$

which conflicts with the optimality of $u^{\Omega^{\prime}, g^{\prime}}$ to $\left(\mathcal{P}^{\Omega^{\prime}, g^{\prime}}\right)$. Therefore, $\left.u^{\Omega^{\prime}, g^{\prime}}\right|_{\Omega^{\prime \prime}}$ is the unique optimal solution to $\left(\mathcal{P}^{\Omega^{\prime \prime}, g^{\prime \prime}}\right)$.

The second consequence of the uniqueness assumption $\left(\mathcal{A}_{U}\right)$ is that the optimal solution $u_{r}^{\Omega, g}$ to the obstacle problem $\left(\mathcal{P}_{r}^{\Omega, g}\right)$ equals the optimal solution $u^{\Omega^{\prime}, g^{\prime}}$ to the problem without obstacle $\left(\mathcal{P}^{\Omega^{\prime}, g^{\prime}}\right)$ in such subdomains where they are coincided on the boundary and $u^{\Omega^{\prime}}, g^{\prime}$ does not violate the obstacle condition, as the following says.

Proposition 3.2. Let $u_{r}^{\Omega, g}$ be optimal to $\left(\mathcal{P}_{r}^{\Omega, g}\right)$, and $u^{\Omega^{\prime}, g^{\prime}}$ be optimal to $\left(\mathcal{P}^{\Omega^{\prime}, g^{\prime}}\right)$. Assume $\Omega^{\prime \prime} \subset \Omega^{\prime} \subset \Omega$,

$$
u_{r}^{\Omega, g}=u^{\Omega^{\prime}, g^{\prime}} \quad \text { on } \partial \Omega^{\prime \prime} \quad \text { and } \quad u^{\Omega^{\prime}, g^{\prime}} \geq r \quad \text { in } \Omega^{\prime \prime} .
$$

Then

$$
u_{r}^{\Omega, g}=u^{\Omega^{\prime}, g^{\prime}} \quad \text { in } \quad \Omega^{\prime \prime}
$$

Proof. Assume the contrary that

$$
u_{r}^{\Omega, g} \not \equiv u^{\Omega^{\prime}, g^{\prime}} \text { in } \Omega^{\prime \prime} .
$$

Then, by $\left(\mathcal{V}_{2}\right)-\left(\mathcal{V}_{3}\right)$, the function

$$
u(x):= \begin{cases}u_{r}^{\Omega, g}(x) & \text { if } x \in \Omega^{\prime \prime} \\ u^{\Omega^{\prime}, g^{\prime}}(x) & \text { if } x \in \Omega^{\prime} \backslash \Omega^{\prime \prime}\end{cases}
$$


belongs to $\mathcal{V}\left(\Omega^{\prime}\right)$ and fulfills $u=g^{\prime}$ on $\partial \Omega^{\prime}$, that means, by $(2.4), u \in \mathcal{K}^{\Omega^{\prime}, g^{\prime}}$, but it differs from $u^{\Omega^{\prime}, g^{\prime}}$ within $\Omega^{\prime}$. Therefore, assumption $\left(\mathcal{A}_{U}\right)$ yields

$$
\begin{aligned}
0 & <\mathcal{F}^{\Omega^{\prime}}(u)-\mathcal{F}^{\Omega^{\prime}}\left(u^{\Omega^{\prime}, g^{\prime}}\right) \\
& =\mathcal{F}^{\Omega^{\prime \prime}}(u)-\mathcal{F}^{\Omega^{\prime \prime}}\left(u^{\Omega^{\prime}, g^{\prime}}\right)+\mathcal{F}^{\Omega^{\prime} \backslash \Omega^{\prime \prime}}(u)-\mathcal{F}^{\Omega^{\prime} \backslash \Omega^{\prime \prime}}\left(u^{\Omega^{\prime}, g^{\prime}}\right) \\
& =\mathcal{F}^{\Omega^{\prime \prime}}\left(u_{r}^{\Omega, g}\right)-\mathcal{F}^{\Omega^{\prime \prime}}\left(u^{\Omega^{\prime}, g^{\prime}}\right) .
\end{aligned}
$$

Consider

$$
\tilde{u}(x):= \begin{cases}u^{\Omega^{\prime}, g^{\prime}}(x) & \text { if } x \in \Omega^{\prime \prime} \\ u_{r}^{\Omega, g}(x) & \text { if } x \in \Omega \backslash \Omega^{\prime \prime} .\end{cases}
$$

By $\left(\mathcal{V}_{2}\right)-\left(\mathcal{V}_{3}\right)$, we have $\tilde{u} \in \mathcal{V}(\Omega)$. Furthermore, it follows from $u_{r}^{\Omega, g} \in \mathcal{K}_{r}^{\Omega, g}$ and (3.1) that

$$
\tilde{u} \geq r \text { in } \Omega \quad \text { and } \quad \tilde{u}=g \text { on } \partial \Omega \text {, }
$$

that means, by (2.1), $\tilde{u} \in \mathcal{K}_{r}^{\Omega, g}$. Continuing the previous calculation, we have now

$$
\begin{aligned}
0 & <\mathcal{F}^{\Omega^{\prime \prime}}\left(u_{r}^{\Omega, g}\right)-\mathcal{F}^{\Omega^{\prime \prime}}\left(u^{\Omega^{\prime}, g^{\prime}}\right) \\
& =\mathcal{F}^{\Omega^{\prime \prime}}\left(u_{r}^{\Omega, g}\right)-\mathcal{F}^{\Omega^{\prime \prime}}(\tilde{u})+\mathcal{F}^{\Omega \backslash \Omega^{\prime \prime}}\left(u_{r}^{\Omega, g}\right)-\mathcal{F}^{\Omega \backslash \Omega^{\prime \prime}}(\tilde{u}) \\
& =\mathcal{F}^{\Omega}\left(u_{r}^{\Omega, g}\right)-\mathcal{F}^{\Omega}(\tilde{u})
\end{aligned}
$$

That means $\tilde{u}$ is admissible and better than $u_{r}^{\Omega, g}$, a contradiction. Therefore, (3.2) must be true.

The previous proposition is is a basic tool for determining coincidence and noncoincidence sets of optimal solutions to obstacle problems.

Definition 3.1. If $u^{\Omega^{\prime}, g^{\prime}} \in \mathcal{K}^{\Omega^{\prime}, g^{\prime}}$ is optimal to $\left(\mathcal{P}^{\Omega^{\prime}, g^{\prime}}\right)$, where $\Omega^{\prime} \subset \Omega$ and

$$
g^{\prime}(x) \begin{cases}\leq g(x) & \text { if } x \in \partial \Omega^{\prime} \cap \partial \Omega \\ =r(x) & \text { if } x \in \partial \Omega^{\prime} \cap \Omega,\end{cases}
$$

then it is named a lower barrier.

The reason for calling such a function as a lower barrier is given in the following.

Proposition 3.3. Let $u_{r}^{\Omega, g}$ be optimal to $\left(\mathcal{P}_{r}^{\Omega, g}\right)$, and $u^{\Omega^{\prime}, g^{\prime}}$ be a lower barrier. Then

$$
u_{r}^{\Omega, g} \geq u^{\Omega^{\prime}, g^{\prime}} \quad \text { in } \quad \Omega^{\prime} .
$$

Proof. Assume the contrary that there exists some $x \in \Omega^{\prime}$ such that $u_{r}^{\Omega, g}(x)<u^{\Omega^{\prime}, g^{\prime}}(x)$. Since

$$
u_{r}^{\Omega, g}=g \text { on } \partial \Omega \quad \text { and } \quad u_{r}^{\Omega, g} \geq r \text { in } \Omega
$$


and

$$
u^{\Omega^{\prime}, g^{\prime}}=g^{\prime} \text { on } \partial \Omega^{\prime}
$$

it follows from (3.3) and the continuity of $u_{r}^{\Omega, g}$ and $u^{\Omega^{\prime}, g^{\prime}}$ that there exists a subdomain $\Omega^{\prime \prime}$ with

$$
x \in \Omega^{\prime \prime} \subset \Omega^{\prime}
$$

and

$$
u_{r}^{\Omega, g}=u^{\Omega^{\prime}, g^{\prime}} \text { on } \partial \Omega^{\prime \prime} \text { and } r \leq u_{r}^{\Omega, g}<u^{\Omega^{\prime}, g^{\prime}} \text { in } \Omega^{\prime \prime} .
$$

Therefore, Proposition 3.2 yields

$$
u_{r}^{\Omega, g}=u^{\Omega^{\prime}, g^{\prime}} \quad \text { in } \quad \Omega^{\prime \prime}
$$

which conflicts with $x \in \Omega^{\prime \prime}$ and $u_{r}^{\Omega, g}(x)<u^{\Omega^{\prime}, g^{\prime}}(x)$. Hence, (3.4) must be true.

The preceding result is useful for finding subsets of noncoincidence points. For instance, for an arbitrary subdomain $\Omega^{\prime} \subset \Omega$, just choose

$$
g^{\prime}=r \quad \text { on } \quad \partial \Omega^{\prime}
$$

and consider the optimal solution to $\left(\mathcal{P}^{\Omega^{\prime}, g^{\prime}}\right)$. By denoting

$$
\mathcal{L}^{+}(v):=\{x \in \Omega: v(x)>r(x)\}
$$

we have

Corollary 3.4. Assume $\Omega^{\prime} \subset \Omega$ and (3.5). Let $u_{r}^{\Omega, g}$ be optimal to $\left(\mathcal{P}_{r}^{\Omega, g}\right)$ and $u^{\Omega^{\prime}, g^{\prime}}$ be optimal to $\left(\mathcal{P}^{\Omega^{\prime}, g^{\prime}}\right)$. Then it holds for the noncoincidence set

$$
\mathcal{L}^{+}\left(u^{\Omega^{\prime}, g^{\prime}}\right) \subset \mathcal{N}\left(u_{r}^{\Omega, g}\right)
$$

Proof. Since $g \geq r$ on $\partial \Omega$, (3.3) follows from (3.5). Therefore, $u^{\Omega^{\prime}, g^{\prime}}$ is a lower barrier, and by Proposition 3.3,

$$
u_{r}^{\Omega, g}(x) \geq u^{\Omega^{\prime}, g^{\prime}}(x)>r(x) \quad \text { for } \quad x \in \mathcal{L}^{+}\left(u^{\Omega^{\prime}, g^{\prime}}\right),
$$

that means (3.7) is fulfilled.

An interesting special case is $\Omega^{\prime}=\Omega$ and $g^{\prime}=g$. By definition, $u^{\Omega, g}$ is a lower barrier. Hence $(2.3),(3.4)$ and (3.6) yield immediately Then

Corollary 3.5. Let $u_{r}^{\Omega, g}$ be optimal to $\left(\mathcal{P}_{r}^{\Omega, g}\right)$ and $u^{\Omega, g}$ be optimal to $\left(\mathcal{P}^{\Omega, g}\right)$.

$$
u_{r}^{\Omega, g} \geq u^{\Omega, g} \quad \text { in } \quad \Omega
$$


and

$$
\mathcal{L}^{+}\left(u^{\Omega, g}\right) \subset \mathcal{N}\left(u_{r}^{\Omega, g}\right)
$$

Definition 3.2. The optimal solution $u^{\Omega, g^{\prime}}$ to $\left(\mathcal{P}^{\Omega, g^{\prime}}\right)$ is said to be an upper barrier provided

$$
g^{\prime}(x) \geq g(x) \quad \text { on } \quad \partial \Omega
$$

and

$$
u^{\Omega, g^{\prime}} \geq r \quad \text { in } \quad \Omega \text {. }
$$

Proposition 3.6. Let $u_{r}^{\Omega, g}$ be optimal to $\left(\mathcal{P}_{r}^{\Omega, g}\right)$, and $u^{\Omega, g^{\prime}}$ be an upper barrier. Then

$$
u_{r}^{\Omega, g} \leq u^{\Omega, g^{\prime}} \quad \text { in } \quad \Omega \text {. }
$$

Proof. Assume the contrary that there is an $x \in \Omega$ such that

$$
u_{r}^{\Omega, g}(x)>u^{\Omega, g^{\prime}}(x) .
$$

Since $u_{r}^{\Omega, g}=g$ and $u^{\Omega, g^{\prime}}=g^{\prime}$ on $\partial \Omega$, it follows from (3.8) and the continuity of $u_{r}^{\Omega, g}$ and $u^{\Omega, g^{\prime}}$ that there exists a subdomain $\Omega^{\prime} \subset \Omega$ such that

$$
x \in \Omega^{\prime} \quad \text { and } \quad u_{r}^{\Omega, g}=u^{\Omega, g^{\prime}} \quad \text { on } \quad \partial \Omega^{\prime} .
$$

Since $u^{\Omega, g^{\prime}} \geq r$ in $\Omega^{\prime}$, Proposition 3.2 yields

$$
u_{r}^{\Omega, g}=u^{\Omega, g^{\prime}} \quad \text { in } \quad \Omega^{\prime}
$$

which conflicts with $x \in \Omega^{\prime}$ and (3.11). Therefore, (3.10) must be true.

Definition 3.3. $x^{*} \in \Omega$ is called a bottle-neck point provided there exists an upper barrier $u^{\Omega, g^{\prime}}$ satisfying $u^{\Omega, g^{\prime}}\left(x^{*}\right)=r\left(x^{*}\right)$.

Proposition 3.7. Let $u_{r}^{\Omega, g}$ be optimal to $\left(\mathcal{P}_{r}^{\Omega, g}\right)$, and $x^{*}$ be a bottle-neck point. Then $x^{*} \in \mathcal{C}\left(u_{r}^{\Omega, g}\right)$, i.e.

$$
u_{r}^{\Omega, g}\left(x^{*}\right)=r\left(x^{*}\right)
$$

Proof. By definition, there exists an upper barrier $u^{\Omega, g^{\prime}}$ satisfying

$$
r\left(x^{*}\right)=u^{\Omega, g^{\prime}}\left(x^{*}\right)
$$

Proposition 3.6 implies

$$
u^{\Omega, g^{\prime}}\left(x^{*}\right) \geq u_{r}^{\Omega, g}\left(x^{*}\right) .
$$


Since $u_{r}^{\Omega, g}\left(x^{*}\right) \geq r\left(x^{*}\right)$, it follows immediately $u_{r}^{\Omega, g}\left(x^{*}\right)=r\left(x^{*}\right)$.

Proposition 3.8. Let $u_{r}^{\Omega, g}$ be optimal to $\left(\mathcal{P}_{r}^{\Omega, g}\right)$ and $u^{\Omega, g^{\prime}}$ be optimal to $\left(\mathcal{P}^{\Omega, g^{\prime}}\right)$, where

$$
g^{\prime} \geq g \quad \text { on } \partial \Omega \quad \text { and } \quad u^{\Omega, g^{\prime}} \geq r \text { in } \Omega .
$$

Assume that there is a subdomain $\Omega^{\prime \prime} \subset \Omega$ such that

$$
u^{\Omega, g^{\prime}}= \begin{cases}g & \text { on } \partial \Omega^{\prime \prime} \cap \partial \Omega \\ r & \text { on } \partial \Omega^{\prime \prime} \cap \Omega\end{cases}
$$

Then

$$
u_{r}^{\Omega, g}=u^{\Omega, g^{\prime}} \quad \text { in } \quad \Omega^{\prime \prime} .
$$

Proof. By definition and (3.12), $u^{\Omega, g^{\prime}}$ is an upper barrier. Thus Proposition 3.6 yields

$$
u_{r}^{\Omega, g} \leq u^{\Omega, g^{\prime}} \quad \text { in } \quad \Omega .
$$

On the other hand, it follows from definition and (3.13) that the restriction of $u^{\Omega, g^{\prime}}$ on $\Omega^{\prime \prime}$ is a lower barrier. Therefore, Proposition 3.3 implies

$$
u_{r}^{\Omega, g} \geq u^{\Omega, g^{\prime}} \text { in } \quad \Omega^{\prime \prime} .
$$

Combining this with (3.15), we obtain (3.14) at once.

\section{Examples of Use}

Throughout this section we assume that $r$ is continuous on $\bar{\Omega}$.

To illustrate the applicability of the result of the previous section, we now consider a special case, where the following invariance assumption is made:

$\left(\mathcal{A}_{I}\right)$ It holds for every $\Omega^{\prime} \subset \Omega$ and $m \in \mathbb{R}$

$$
\mathcal{F}^{\Omega^{\prime}}(v+m)-\mathcal{F}^{\Omega^{\prime}}(v)=\text { const }=: c^{\Omega^{\prime}}(m) \text { for all } v \in \mathcal{V}\left(\Omega^{\prime}\right) .
$$

Of course, this is a strong restriction. But numerous relevant problems satisfy it. For instance, for the performance indexes in Examples 2.1-2.4 we have

$$
\mathcal{F}_{1}^{\Omega^{\prime}}(v+m)-\mathcal{F}_{1}^{\Omega^{\prime}}(v)=\mathcal{F}_{2}^{\Omega^{\prime}}(v+m)-\mathcal{F}_{2}^{\Omega^{\prime}}(v)=0
$$

and

$$
\mathcal{F}_{3}^{\Omega^{\prime}}(v+m)-\mathcal{F}_{3}^{\Omega^{\prime}}(v)=\mathcal{F}_{6}^{\Omega^{\prime}}(v+m)-\mathcal{F}_{6}^{\Omega^{\prime}}(v)=m \int_{\Omega^{\prime}} f d x=\text { const }
$$


for all $v \in \mathcal{V}\left(\Omega^{\prime}\right)$.

In general, if $F(v)$ contains only derivatives of $v$, such as $\nabla v$ and $\triangle v$, or $v$ appears explicitly only affinely there, then $\left(\mathcal{A}_{I}\right)$ is fulfilled.

Actually, $\left(\mathcal{A}_{I}\right)$ belongs to such assumptions which ensure the continuous dependence of the optimal solution $u^{\Omega^{\prime}, g^{\prime}}$ to Problem $\left(\mathcal{P}^{\Omega^{\prime}, g^{\prime}}\right)$ on the parameter $g^{\prime}$. Moreover, it allows vertical movement without changing the optimal shape, as the following says.

Proposition 4.1. Let $u^{\Omega^{\prime}, g^{\prime}}$ be optimal to $\left(\mathcal{P}^{\Omega^{\prime}, g^{\prime}}\right)$. Then, for arbitrary $m \in \mathbb{R}, u^{\Omega^{\prime}, g^{\prime}}+m$ is optimal to $\left(\mathcal{P}^{\Omega^{\prime}, g^{\prime}+m}\right)$.

Proof. (4.1) implies

$$
\mathcal{F}^{\Omega^{\prime}}\left(u^{\Omega^{\prime}, g^{\prime}}+m\right)=\mathcal{F}^{\Omega^{\prime}}\left(u^{\Omega^{\prime}, g^{\prime}}\right)+c^{\Omega^{\prime}}(m) \leq \mathcal{F}^{\Omega^{\prime}}(v)+c^{\Omega^{\prime}}(m)=\mathcal{F}^{\Omega^{\prime}}(v+m)
$$

for all $v \in \mathcal{K}^{\Omega^{\prime}, g^{\prime}}$. But by $(2.4)$,

$$
v \in \mathcal{K}^{\Omega^{\prime}, g^{\prime}} \quad \text { iff } \quad v+m \in \mathcal{K}^{\Omega^{\prime}, g^{\prime}+m}
$$

Therefore, we have $u^{\Omega^{\prime}, g^{\prime}}+m \in \mathcal{K}^{\Omega^{\prime}, g^{\prime}+m}$ and

$$
\mathcal{F}^{\Omega^{\prime}}\left(u^{\Omega^{\prime}, g^{\prime}}+m\right) \leq \mathcal{F}^{\Omega^{\prime}}(w) \text { for all } w \in \mathcal{K}^{\Omega^{\prime}, g^{\prime}+m}
$$

that means $u^{\Omega^{\prime}, g^{\prime}}+m$ is optimal to $\left(\mathcal{P}^{\Omega^{\prime}, g^{\prime}+m}\right)$.

The above property can be applied to determine some parts of noncoincidence set.

Proposition 4.2. Assume $u^{\Omega^{\prime}, g^{\prime}}$ is optimal to $\left(\mathcal{P}^{\Omega^{\prime}, g^{\prime}}\right), \Omega^{\prime \prime} \subset \Omega^{\prime}$ is an open subset, and

$$
u^{\Omega^{\prime}, g^{\prime}}(x)-r(x)>m=u^{\Omega^{\prime}, g^{\prime}}(y)-r(y) \quad \text { for all } \quad x \in \Omega^{\prime \prime}, y \in \partial \Omega^{\prime \prime} .
$$

Then $\Omega^{\prime \prime}$ is a subset of the noncoincidence set $\mathcal{N}\left(u_{r}^{\Omega, g}\right)$.

Proof. By Proposition 4.1, $u^{\Omega^{\prime}, g^{\prime}}-m$ is optimal to $\left(\mathcal{P}^{\Omega^{\prime}, g^{\prime}-m}\right)$. By assumption, we have $u^{\Omega^{\prime}, g^{\prime}}-m=r$ on $\partial \Omega^{\prime \prime}$. Therefore, the restriction of $u^{\Omega^{\prime}, g^{\prime}}-m$ on $\Omega^{\prime \prime}$ is a lower barrier, and Corollary 3.4 yields

$$
\Omega^{\prime \prime} \cap \mathcal{L}^{+}\left(u^{\Omega^{\prime}, g^{\prime}}-m\right) \subset \mathcal{N}\left(u_{r}^{\Omega, g}\right)
$$

Since $\Omega^{\prime \prime} \subset \mathcal{L}^{+}\left(u^{\Omega^{\prime}, g^{\prime}}-m\right)$, we have $\Omega^{\prime \prime} \subset \mathcal{N}\left(u_{r}^{\Omega, g}\right)$.

Mention that $\Omega^{\prime \prime}$ given in Proposition 4.2 is an open subset. It is a bit more difficult to ensure a closed subset to be contained in the noncoincidence set. For this purpose, we need the following notion. 
Definition 4.1. A closed subset $A \subset \Omega$ is said to be a locally strictly maximal region of $v$ provided there exists an open subset $B \subset \Omega$ which contains $A$ and satisfies

$$
v(x)>v(y) \quad \text { for all } \quad x \in A, y \in B \backslash A .
$$

It follows from $(4.2)$

$$
\inf _{x \in \partial A} v(x) \geq \inf _{x \in A} v(x) \geq \sup _{x \in B \backslash A} v(x) .
$$

The continuity of $v$ yields

$$
\sup _{x \in B \backslash A} v(x)=\sup _{x \in(B \backslash A) \cup \partial A} v(x) \geq \sup _{x \in \partial A} v(x) .
$$

Hence $v=$ const on $\partial A$. Therefore, for continuous $v,(4.2)$ is equivalent to

$$
v \equiv a \text { on } \partial A \quad \text { and } \quad v(x) \geq a>v(y) \text { for all } x \in A, y \in B \backslash A
$$

(for some constant $a$ ).

Proposition 4.3. Let $u^{\Omega^{\prime}, g^{\prime}}$ be optimal to $\left(\mathcal{P}^{\Omega^{\prime}, g^{\prime}}\right)$ for some bounded $\Omega^{\prime} \subset \Omega$ and some continuous function $g^{\prime}$ on $\partial \Omega^{\prime}$, and $u_{r}^{\Omega, g}$ be optimal to $\left(\mathcal{P}_{r}^{\Omega, g}\right)$. Assume $A \subset \Omega^{\prime}$ is a locally strictly maximal region of $u^{\Omega^{\prime}, g^{\prime}}-r$, then $A$ is a subset of the noncoincidence set $\mathcal{N}\left(u_{r}^{\Omega, g}\right)$.

Proof. By definition, there exists an open subset $B$ satisfying $A \subset B \subset$ $\Omega^{\prime} \subset \Omega$ and (4.2). Take an arbitrary open subset $B^{\prime}$ with

$$
A \subset B^{\prime} \subset \overline{B^{\prime}} \subset B
$$

Since $\partial B^{\prime}$ is compact and $u^{\Omega^{\prime}, g^{\prime}}-r$ is continuous on $\overline{\Omega^{\prime}}$, there exists $x^{*} \in \partial B^{\prime}$ such that

$$
u^{\Omega^{\prime}, g^{\prime}}\left(x^{*}\right)-r\left(x^{*}\right)=m:=\max _{x \in \partial B^{\prime}}\left(u^{\Omega^{\prime}, g^{\prime}}(x)-r(x)\right) .
$$

Consider

$$
\Omega^{\prime \prime}:=\left\{x \in B^{\prime}: u^{\Omega^{\prime}, g^{\prime}}(x)-r(x)>m\right\} .
$$

(4.4) yields $x^{*} \in \partial B^{\prime} \subset B \backslash A$. Therefore, (4.2) implies

$$
u^{\Omega^{\prime}, g^{\prime}}(x)-r(x)>u^{\Omega^{\prime}, g^{\prime}}\left(x^{*}\right)-r\left(x^{*}\right)=m \text { for all } x \in A,
$$

that means $A \subset \Omega^{\prime \prime}$. Moreover, since $\overline{\Omega^{\prime \prime}} \subset \overline{B^{\prime}} \subset B$ and $u^{\Omega^{\prime}, g^{\prime}}-r$ is continuous, it follows that $\Omega^{\prime \prime}$ is open and

$$
u^{\Omega^{\prime}, g^{\prime}}(x)-r(x)=m \quad \text { on } \quad x \in \partial \Omega^{\prime \prime} .
$$


Assume now that $\Omega^{\prime \prime}$ is connected, i.e. it is a subdomain of $\Omega$. Proposition 3.1 implies that the restriction $\left.u^{\Omega^{\prime}, g^{\prime}}\right|_{\Omega^{\prime \prime}}$ is optimal to $\left(\mathcal{P}^{\Omega^{\prime \prime}, g^{\prime \prime}}\right)$ with $g^{\prime \prime}=\left.u^{\Omega^{\prime}, g^{\prime}}\right|_{\partial \Omega^{\prime \prime}}$. Hence, by Proposition 4.1, the restriction of $u^{\Omega^{\prime}, g^{\prime}}-m$ on $\Omega^{\prime \prime}$ is optimal to $\left(\mathcal{P}^{\Omega^{\prime \prime}, g^{\prime \prime}-m}\right)$. By (4.6), we have $u^{\Omega^{\prime}, g^{\prime}}-m=r$ on $\partial \Omega^{\prime \prime}$. Therefore, the restriction of $u^{\Omega^{\prime}, g^{\prime}}-m$ on $\Omega^{\prime \prime}$ is a lower barrier, and Corollary 3.4 yields

$$
\Omega^{\prime \prime} \cap \mathcal{L}^{+}\left(u^{\Omega^{\prime}, g^{\prime}}-m\right) \subset \mathcal{N}\left(u_{r}^{\Omega, g}\right) .
$$

Since $A \subset \Omega^{\prime \prime} \cap \mathcal{L}^{+}\left(u^{\Omega^{\prime}, g^{\prime}}-m\right)$ follows from (4.5), it holds

$$
A \subset \mathcal{N}\left(u_{r}^{\Omega, g}\right)
$$

If $\Omega^{\prime \prime}$ is not connected, we only have to deal with each component of $\Omega^{\prime \prime}$ which contains some part of $A$ in the same way as above to obtain the desired conclusion.

In particular, if it holds for the optimal solution $u^{\Omega^{\prime}, g^{\prime}}$ to some $\left(\mathcal{P}^{\Omega^{\prime}, g^{\prime}}\right)$

$$
u^{\Omega^{\prime}, g^{\prime}}\left(x^{*}\right)-r\left(x^{*}\right)>u^{\Omega^{\prime}, g^{\prime}}(x)-r(x) \text { for } x \in B\left(x^{*}, \varepsilon\right) \backslash\left\{x^{*}\right\} \subset \Omega^{\prime}
$$

(for some $\varepsilon>0$ ), that means (4.2) is valid for $A=\left\{x^{*}\right\}$, then Proposition 4.3 ensures $x^{*} \in \mathcal{N}\left(u_{r}^{\Omega, g}\right)$, that means $u_{r}^{\Omega, g}\left(x^{*}\right)=r\left(x^{*}\right)$.

By the propositions stated above we can locate the noncoincidence set $\mathcal{N}\left(u_{r}^{\Omega, g}\right)$. This technique is an outer approach to the coincidence set $\mathcal{C}\left(u_{r}^{\Omega, g}\right)$. Let us state a direct approach to the coincidence set now.

Proposition 4.4. Let $u^{\Omega, g^{\prime}}$ be optimal to $\left(\mathcal{P}^{\Omega, g^{\prime}}\right)$ for some continuous function $g^{\prime}$ on $\partial \Omega$ satisfying $g^{\prime} \geq g$, and $u_{r}^{\Omega, g}$ be optimal to $\left(\mathcal{P}_{r}^{\Omega, g}\right)$. Assume $x^{*} \in \Omega$ and

$$
u^{\Omega, g^{\prime}}\left(x^{*}\right)-r\left(x^{*}\right)=\min _{x \in \Omega}\left(u^{\Omega, g^{\prime}}(x)-r(x)\right) \leq 0 .
$$

Then $x^{*} \in \mathcal{C}\left(u_{r}^{\Omega, g}\right)$, that means $u_{r}^{\Omega, g}\left(x^{*}\right)=r\left(x^{*}\right)$.

Proof. Denote $m=u^{\Omega, g^{\prime}}\left(x^{*}\right)-r\left(x^{*}\right) \leq 0$. By Proposition 4.1, $u^{\Omega, g^{\prime}}-m$ is optimal to $\left(\mathcal{P}^{\Omega, g^{\prime}-m}\right)$. (4.7) implies

$$
g^{\prime}-m \geq g^{\prime} \geq g \text { on } \partial \Omega \quad \text { and } \quad u^{\Omega, g^{\prime}}(x)-m \geq r(x) \text { in } \Omega,
$$

that means $u^{\Omega, g^{\prime}}-m$ is an upper barrier. Since $u^{\Omega, g^{\prime}}\left(x^{*}\right)-m=r\left(x^{*}\right), x^{*}$ is a bottle-neck point. Therefore, Proposition 3.7 yields $x^{*} \in \mathcal{C}\left(u_{r}^{\Omega, g}\right)$.

To illustrate easily the conclusions of this paper, let us consider the following simple problem.

Example 4.1.

$$
\begin{array}{cl}
\operatorname{minimize} & \mathcal{F}_{8}^{(-2,3)}(v):=\int_{-2}^{3}\left(2 v+\left|v^{\prime}\right|^{2}\right) d x \\
\text { subject to } & v \in W^{1,2}(-2,3), v \geq r(x)=|x| \text { in }(-2,3), \\
& v(-2)=4, v(3)=5
\end{array}
$$


For $\Omega \subset \mathbb{R}$, the embedding $W^{1,2}(\Omega) \subset C(\bar{\Omega})$ is compact (see [16, p. 1027]), therefore $\left(\mathcal{V}_{1}\right)-\left(\mathcal{V}_{3}\right)$ are satisfied for $\mathcal{V}(\Omega)=W^{1,2}(\Omega)$. Since (4.8) is only a special case of the problem considered in Example 2.3 when $\mathcal{F}_{4}^{\Omega}(v)$ is approximated by $\mathcal{F}_{1}^{\Omega}(v),\left(\mathcal{A}_{U}\right)$ is fulfilled.

Consider the auxiliary problem

$$
\begin{array}{ll}
\text { minimize } & \mathcal{F}_{8}^{\left(t_{a}, t_{b}\right)}(v)=\int_{t_{a}}^{t_{b}}\left(2 v+\left|v^{\prime}\right|^{2}\right) d x \\
\text { subject to } & v \in W^{1,2}\left(t_{a}, t_{b}\right), \quad v\left(t_{a}\right)=a, \quad v\left(t_{b}\right)=b,
\end{array}
$$

where $\Omega^{\prime}=\left(t_{a}, t_{b}\right) \subset(-2,3) \subset \mathbb{R}$. $\left(\mathcal{A}_{I}\right)$ is satisfied, because

$$
\mathcal{F}_{8}^{\left(t_{a}, t_{b}\right)}(v+m)-\mathcal{F}_{8}^{\left(t_{a}, t_{b}\right)}(v)=2 m\left(t_{b}-t_{a}\right)=\mathrm{const} \quad \text { for all } v \in W^{1,2}\left(t_{a}, t_{b}\right) .
$$

The corresponding Euler equation

$$
1-v^{\prime \prime}=0
$$

yields

$$
v(x)=x^{2}+c x+d
$$

for some $c, d \in \mathbb{R}$. Combining with the boundary condition

$$
v=g^{\prime} \quad \text { on } \quad \partial \Omega^{\prime}, \quad \text { where } \quad g^{\prime}(x):= \begin{cases}a & \text { for } x=t_{a} \\ b & \text { for } x=t_{b}\end{cases}
$$

we obtain

$$
u^{\Omega^{\prime}, g^{\prime}}(x):=x^{2}+c x+d, \quad \text { where } \quad c=\frac{b-a}{t_{b}-t_{a}}-t_{a}-t_{b}, d=\frac{a t_{b}-b t_{a}}{t_{b}-t_{a}}+t_{a} t_{b},
$$

satisfying the Euler equation (4.10) and the boundary condition (4.11). Moreover, 0 is the Gateaux derivative of the convex functional $\mathcal{F}_{8}^{\left(t_{a}, t_{b}\right)}$ at $u^{\Omega^{\prime}, g^{\prime}}$, therefore

$$
0 \in \partial \mathcal{F}_{8}^{\left(t_{a}, t_{b}\right)}\left(u^{\Omega^{\prime}, g^{\prime}}\right)
$$

(see [6, p. 22 and p. 46]), which is sufficient for $u^{\Omega^{\prime}, g^{\prime}}$ to be optimal to Problem $\left(\mathcal{P}^{\Omega^{\prime}, g^{\prime}}\right)$ given by $(4.9)$ (see $[6$, p. 81]).

Let us now apply the conclusions stated above.

(a) Consider $\Omega=(-2,3), g(-2)=4$ and $g(3)=5$. Clearly,

$$
u^{\Omega, g}=x^{2}-0.8 x-1.6
$$


is optimal to $\left(\mathcal{P}^{\Omega, g}\right)$. By Definition 3.1, $u^{\Omega, g}$ is a lower barrier. Therefore, Proposition 3.3 yields for the optimal solution $u_{r}^{\Omega, g}$ to Problem $\left(\mathcal{P}_{r}^{\Omega, g}\right)$ given by (4.8) that

$$
u_{r}^{\Omega, g}(x) \geq x^{2}-0.8 x-1.6 .
$$

Since

$$
\begin{aligned}
\mathcal{L}^{+}\left(u_{r}^{\Omega, g}\right) & =\left\{x \in(-2,3): x^{2}-0.8 x-1.6-|x|>0\right\} \\
& =(-2,-0.1-\sqrt{1.61}) \cup(0.9+\sqrt{2.41}, 3),
\end{aligned}
$$

Corollary 3.4 implies

$$
(-2,-0.1-\sqrt{1.61}) \cup(0.9+\sqrt{2.41}, 3) \subset \mathcal{N}\left(u_{r}^{\Omega, g}\right) .
$$

(b) For

$$
z \in[-2+\sqrt{2},-0.5], \quad g^{\prime}(-2)=z^{2}+4 z+6 \quad \text { and } \quad g^{\prime}(3)=z^{2}-6 z+6
$$

we can show that

$$
u^{\Omega, g^{\prime}}(x)=x^{2}-(1+2 z) x+z^{2}
$$

is optimal to $\left(\mathcal{P}^{\Omega, g^{\prime}}\right)$ and satisfies (3.8)-(3.9). Therefore, by Definition 3.2, $u^{\Omega, g^{\prime}}$ is an upper barrier, that yields by Proposition 3.6

$$
u_{r}^{\Omega, g}(x) \leq x^{2}-(1+2 z) x+z^{2} .
$$

Moreover, since

$$
u^{\Omega, g}(z)=-z=|z|=r(z),
$$

according to Definition 3.3, $z$ is a bottle-neck point. Hence, Proposition 3.7 implies

$$
u_{r}^{\Omega, g}(z)=r(z)=-z \quad \text { for } \quad z \in[-2+\sqrt{2},-0.5],
$$

that means

$$
[-2+\sqrt{2},-0.5] \subset \mathcal{C}\left(u_{r}^{\Omega, g}\right) .
$$

(c) With $\Omega=(-2,3), g^{\prime}(-2)=4.25$ and $g^{\prime}(3)=9.25$, we have

$$
\begin{aligned}
& u^{\Omega, g^{\prime}}(x)=x^{2}+0.25 \geq r(x)=|x| \text { for } \quad-2 \leq x \leq 3, \\
& u^{\Omega, g^{\prime}}( \pm 0.5)=0.5=| \pm 0.5|=r( \pm 0.5),
\end{aligned}
$$

i.e., (3.12)-(3.13) hold for $\Omega^{\prime \prime}=(-0.5,0.5)$. Consequently, Proposition 3.8 implies

$$
u_{r}^{\Omega, g}(x)=x^{2}+0.25 \quad \text { for } \quad|x| \leq 0.5 .
$$


(d) Assume now $\Omega^{\prime}=\Omega=(-2,3), g^{\prime}(-2)=4$ and $g^{\prime}(3)=9$. Then

$$
u^{\Omega^{\prime}, g^{\prime}}(x)=x^{2}
$$

is optimal to $\left(\mathcal{P}^{\Omega^{\prime}, g^{\prime}}\right)$. Since

$$
\frac{d}{d x}\left(u^{\Omega^{\prime}, g^{\prime}}(x)-r(x)\right)=\frac{d}{d x}\left(x^{2}-|x|\right) \begin{cases}>0 & \text { if }-0.5<x<0 \\ <0 & \text { if } 0<x<0.5\end{cases}
$$

the assumption of Proposition 4.3 is fulfilled for every closed interval $[-\alpha, \alpha]$ contained in $(-0.5,0.5)$. Therefore, this proposition yields $[-\alpha, \alpha] \subset \mathcal{N}\left(u_{r}^{\Omega, g}\right)$ for $|\alpha|<$ 0.5. Actually, the assumption of Proposition 4.2 is satisfied for $\Omega^{\prime \prime}=(-0.5,0.5)$. Hence,

$$
(-0.5,0.5) \subset \mathcal{N}\left(u_{r}^{\Omega, g}\right)
$$

follows directly from Proposition 4.2. Since $u^{\Omega^{\prime}, g^{\prime}}-r$ attains its global minimum at $x= \pm 0.5$ and

$$
u^{\Omega^{\prime}, g^{\prime}}( \pm 0.5)-r( \pm 0.5)=-0.25<0,
$$

Proposition 4.4 shows that $\pm 0.5 \in \mathcal{C}\left(u_{r}^{\Omega, g}\right)$. Obviously, this result is appropriate to the one in $(c)$.

(e) By choosing $g^{\prime}(3)=g(3)=5$ and varying

$$
g^{\prime}(-2)=10 z-5 \quad \text { for } \quad z \in[0.9,3-\sqrt{2}]
$$

we obtain

$$
u^{\Omega, g^{\prime}}(x)=x^{2}+(1-2 z) x+6 z-7
$$

as optimal solution to $\left(\mathcal{P}^{\Omega, g^{\prime}}\right)$, which satisfies by $z \geq 0.9$

$$
u^{\Omega, g^{\prime}}(-2)=g^{\prime}(-2) \geq 4=g(-2) .
$$

Moreover, for $z \in[0.9,3-\sqrt{2}]$, the function

$$
u^{\Omega, g^{\prime}}(x)-r(x)=x^{2}+(1-2 z) x+6 z-7-|x|, \quad x \in \Omega=(-2,3),
$$

attains its global minimum at $x=z$, and it holds by $z \leq 3-\sqrt{2}$

$$
u^{\Omega, g^{\prime}}(z)-r(z)=-(z-3)^{2}+2 \leq 0 .
$$

Consequently, Proposition 4.4 implies

$$
u_{r}^{\Omega, g}(z)=r(z)=z \quad \text { for } \quad z \in[0.9,3-\sqrt{2}]
$$

that means

$$
[0.9,3-\sqrt{2}] \subset \mathcal{C}\left(u_{r}^{\Omega, g}\right)
$$


(f) Similarly as in $(e)$, by choosing $g^{\prime}(-2)=g(-2)=4$ and varying

$$
g^{\prime}(3)=14-10 z \quad \text { for } \quad z \in[0.5,0.9]
$$

we obtain

$$
u^{\Omega, g^{\prime}}(x)=x^{2}+(1-2 z) x+2(1-2 z)
$$

as optimal solution to $\left(\mathcal{P}^{\Omega, g^{\prime}}\right)$, which satisfies

$$
\begin{aligned}
& u^{\Omega, g^{\prime}}(3)=g^{\prime}(3) \geq 5=g(3), \\
& u^{\Omega, g^{\prime}}(z)-r(z)=-z^{2}-4 z+2 \leq 0 .
\end{aligned}
$$

Moreover, for $z \in[0.5,0.9]$, the function

$$
u^{\Omega, g^{\prime}}(x)-r(x)=x^{2}+(1-2 z) x+2(1-2 z)-|x|, \quad x \in \Omega=(-2,3),
$$

attains its global minimum at $x=z$. Consequently, Proposition 4.4 implies

$$
[0.5,0.9] \subset \mathcal{C}\left(u_{r}^{\Omega, g}\right)
$$

We have seen how the conclusions of this paper can be applied to investigate optimal solutions to obstacle problems. By choosing different $\Omega^{\prime}$ and $g^{\prime}$, it is possible to locate noncoincidence and coincidence points of the optimal solution $u_{r}^{\Omega, g}$ to the obstacle problem $\left(\mathcal{P}_{r}^{\Omega, g}\right)$. It was shown in such a way that

$$
[-2+\sqrt{2},-0.5] \cup[0.5,3-\sqrt{2}] \subset \mathcal{C}\left(u_{r}^{\Omega, g}\right)
$$

Actually, these are already all coincidence points of the optimal solution $u_{r}^{\Omega, g}$ to the obstacle problem (4.8), which can be shown to equal

$$
u_{r}^{\Omega, g}(x)= \begin{cases}x^{2}+(3-2 \sqrt{2}) x+6-4 \sqrt{2} & \text { if } x \in[-2,-2+\sqrt{2}] \\ x^{2}+0.25 & \text { if }|x| \leq 0.5 \\ x^{2}-(5-2 \sqrt{2}) x+11-6 \sqrt{2} & \text { if } x \in[3-\sqrt{2}, 3] \\ |x| & \text { if } x \in[-2+\sqrt{2},-0.5] \cup[0.5,3-\sqrt{2}]\end{cases}
$$

\section{Concluding Remarks}

Our above conclusions hold true for every optimal solution $u_{r}^{\Omega, g}$ to $\left(\mathcal{P}_{r}^{\Omega, g}\right)$. Properly, under some assumption, it can be shown that the optimal solution to $\left(\mathcal{P}_{r}^{\Omega, g}\right)$ is unique, but we do not intend to deal with this aspect here.

For $\Omega \subset \mathbb{R}$, it is possible to develop a constructive method for solving obstacle problems with higher-order derivatives, for which $\left(\mathcal{A}_{U}\right)$ is the main necessary assumption. This is the subject of another paper. 
Acknowledgments. This paper was written while the first author was visiting the Abdus Salam International Centre for Theoretical Physics, Trieste, Italy, as an Associate. A generous grant from the Swedish International Development Cooperation Agency facilitated the visit. He would like to express his sincere gratitude to both bodies.

\section{References}

[1] Courant, R.: Dirichlet's Principle, Conformal Mapping, and Minimal Surfaces. New York: Interscience 1950.

[2] Dacorogna, B.: Direct Methods in the Calculus of Variations. Berlin etc.: Springer-Verlag 1989.

[3] Dinh, N. and Phu, H. X.: Solving a class of regular optimal control problems with state constraints by the method of orienting curves. Optimization 25 (1992), 231-247.

[4] Dinh, N. and Phu, H. X.: Solving a class of optimal control problems which are linear in the control variable by the method of orienting curves. Acta Mathematica Vietnamica 17 (1992), 115-134.

[5] Dinh, N. and Phu, H. X.: The method of orienting curves and its application to an optimal control problem of hydroelectric power plants. Vietnam Journal of Mathematics 20 (1992) 40-53.

[6] A. D. Ioffe and V. M. Tihomirov: Theory of Extremal Problems, Amsterdam etc.: North-Holland Publishing Company 1979.

[7] Landau, L. and Lipchitz, E.: Théorie de l'Élasticité. Moscou: Mir 1967.

[8] Morrey, C. B.: Multiple Integrals in the Calculus of Variations. Berlin etc.: Springer-Verlag 1966.

[9] Phu, H. X.: Zur Lösung einer regulären Aufgabenklasse der optimalen Steuerung im Großen mittels Orientierungskurven. Optimization 18 (1987), 6581.

[10] Phu, H. X.: Zur Lösung eines Zermeloschen Navigationsproblems. Optimization 18 (1987), 225-236.

[11] Phu, H. X.: Ein konstruktives Lösungsverfahren für das Problem des Inpolygons kleinsten Umfangs von J. Steiner. Optimization 18 (1987), 349-359.

[12] Phu, H. X.: Method of orienting curves for solving optimal control problems with state constraints. Numerical Functional Analysis and Optimization 12 (1991), $173-211$.

[13] Phu, H. X., Bock, H. G., and Schlöder, J.: The method of orienting curves and its application for manipulator trajectory planning. Numerical Functional Analysis and Optimization 18 (1997), 213-225. 
[14] Phu, H. X. and Dinh, N.: Some remarks on the method of orienting curves. Numerical Functional Analysis and Optimization 16 (1995), 755-763.

[15] Rodrigues, J.-F.: Obstacle Problems in Mathematical Physics. Amsterdam etc.: Elsevier Science Publishers B.V. 1987.

[16] Zeidler, E.: Nonlinear Functional Analysis and its Applications II. New York etc.: Springer-Verlag 1990. 